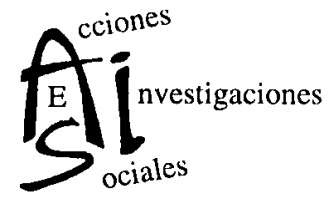

\title{
Regulación jurídica de las pistas de esquí de fondo
}

\author{
José María NasarRe SARMiento \\ Gloria María Hidalgo Rúa \\ DEPARTAMENTO DE DERECHO DE LA EMPRESA \\ UNIVERSIDAD DE ZARAGOZA
}

\section{Pilar Lucia Bernad} ABOGADA

En España no existe regulación de las pistas de esquí de fondo y son pocas las normas legales que se han ocupado de aspectos que tocan mínimamente la organización de las actividades que se concentran en ellas. Sin embargo, pueden apuntarse diferentes instrumentos jurídicos para cubrir el vacío normativo.

Palabras clave: Esquí, esquí de fondo, esquiador, deporte, regulación municipal, ordenanza.

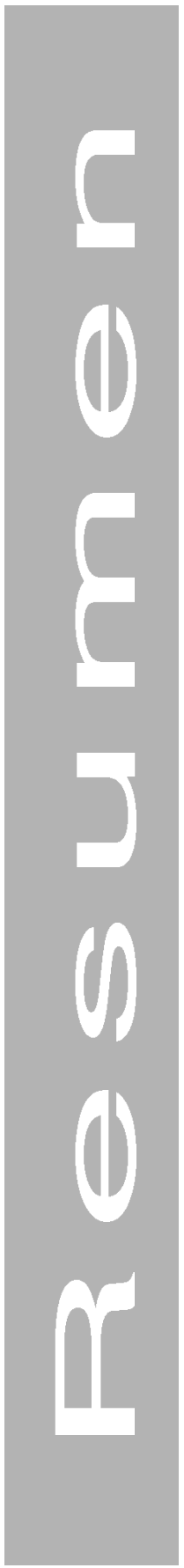




\title{
Regulación jurídica de las pistas de esquí de fondo
}

\author{
José María Nasarre Sarmiento \\ Gloria María Hidalgo Rúa \\ Pilar Lucia Bernad
}

En el año 2000 los autores de este artículo asumimos el estudio de los diferentes aspectos jurídicos que concurrían en las pistas de esquí de fondo de Llanos del Hospital, en el término municipal de Benasque, provincia de Huesca. El Proyecto de Investigación concluyó en el mes de agosto de ese año tras haber analizado tanto las características propias de la relación jurídica entre el Ayuntamiento de Benasque, la empresa concesionaria y el Parque Natural Posets-Maladeta como las posibilidades de ordenación jurídica de una pista municipal de esquí de fondo. Para ciertos casos en los que los accesos, la pista y los edificios de servicios constituyen un bloque homogéneo cuya titularidad es municipal apuntamos la posibilidad de afrontar la regulación mediante ordenanza municipal. Este artículo es fruto de aquellas reflexiones y parece una propuesta válida en la actualidad. Aunque en ninguna comunidad autónoma existe regulación de las pistas de esquí de fondo, pueden utilizarse ciertos instrumentos jurídicos para cubrir el vacío normativo.

\section{Leyes del Deporte}

Aunque no excluye expresamente las pistas de esquí, la Ley 10/1990, del Deporte, no las contempla especialmente al referirse a las instalaciones deportivas, pues no parece tener en cuenta las instalaciones situadas en la naturaleza. De entre las leyes del deporte autonómicas, sólo la Ley 4/1993, de 16 de marzo, del Deporte de Aragón, contiene una mención especí- 
fica, dado que dedica su disposición final tercera a la futura aprobación de un Estatuto que regule las estaciones y centros de esquí y montaña. Los términos empleados siguen las líneas de la Ley de la Montaña francesa.

La Ley 4/1993 establece que en el plazo de un año se remitirá a las Cortes de Aragón un proyecto de ley relativo al Estatuto de las Estaciones y Centros de Esquí y Montaña y advierte que en dicho Estatuto deberán incluirse aspectos como la definición jurídica de las estaciones y centros de esquí y montaña, la responsabilidad, la regulación del dominio esquiable y del transporte por cable, la ordenación urbanística de los centros, las condiciones para el desarrollo de la enseñanza del esquí y de las actividades industriales y de servicios.

Se trata de un planteamiento ciertamente ambicioso, pues el desarrollo de algunos de los puntos habría de ser objeto de un profundo proceso de reflexión colectiva. El debate social que pudiera haberse iniciado entonces no se ha producido, aunque recientemente se empieza a plantear la necesidad de buscar soluciones a los problemas derivados del transporte, aparcamientos, etc, de las estaciones de esquí alpino.

En el año 2002, el Gobierno de Aragón aprobó el Proyecto de Ley de Ordenación, Protección y Desarrollo del Pirineo Aragonés, que trataba de ajustar regulaciones diversas a la singularidad del territorio pirenaico buscando el desarrollo sostenible. Junto a materias tales como la política hidráulica, el urbanismo, la protección ambiental o la comarcalización de Aragón, contemplaba la regulación de los centros de esquí y montaña, que se detallaba de forma acorde con la previsión que había realizado la Ley del Deporte. Esta llamada "Ley del Pirineo" no ha sido aprobada.

\section{La Ley de Ordenación de los Transportes Terrestres}

Al no haberse cumplido las previsiones de la Ley del Deporte de Aragón, la única normativa que afecta a las estaciones de esquí es la establecida en la Ley 16/1987, de 30 de julio, de Ordenación de los Transportes Terrestres, cuya Disposición Adicional $3^{\mathrm{a}}$ se refiere a los medios de transporte de las estaciones de esquí. 
Esta regulación fue ideada para las estaciones de esquí alpino, en las que es necesario el uso de telesillas $u$ otros medios de tracción por cable, que no se precisan para la práctica del esquí de fondo. Sin embargo, a falta de otro tipo de normas, pueden servir de referencia las alusiones que, más allá de lo específico sobre el transporte por cable, se establecen en la Ley y en su Reglamento (Real Decreto 1211/1990, de 28 de Septiembre).

Se considerarán estaciones de invierno o esquí, según la Disposición Adicional 2ª , apartado 1 del Reglamento, aquellos centros turísticos básicamente dedicados a la práctica del esquí y demás deportes de nieve y montaña, que formen un conjunto coordinado de medios de remontes mecánicos, pistas e instalaciones complementarias, de uso público. Se estima que deben reunir unas mínimas condiciones en materias tales como la maquinaria para el acondicionamiento y mantenimiento de las pistas, los suministros de agua y energía eléctrica e instalaciones de saneamiento y eliminación de basuras, el servicio telefónico, los servicios de información general, el puesto de socorro los aparcamientos de vehículos o el personal adecuado.

Aunque no hay ninguna regulación específica que se refiera a las estaciones de esquí de fondo, podrían aplicarse de modo facultativo y parcial, por extensión, las condiciones mínimas exigidas para los centros turísticos dedicados a la práctica del esquí y demás deportes de nieve y montaña.

Muy recientemente, por Decreto 279/2003, de 4 de noviembre, se regulan en Aragón los procedimientos técnicos de explotación y mantenimiento de las instalaciones de transporte por cable en las estaciones de esquí y montaña. Se trata de garantizar la seguridad de los usuarios en el transporte por cable y por tanto no afecta a las estaciones de esquí de fondo.

\section{El reglamento de ATUDEM como intento de solución}

Ante la falta de decisión del legislador español para regular las estaciones de esquí, las estaciones de esquí alpino integradas en Asociación Turística de Estaciones de Esquí y de Montaña (ATUDEM) han redactado su propio reglamento de funcionamiento, a sabiendas de que su valor es muy limitado en 
algunas materias como las concernientes a la seguridad o ineficaz en otras como la responsabilidad del usuario. El reglamento destaca en su preámbulo los dos problemas que causan la máxima preocupación que son la seguridad de las estaciones y la delimitación de las responsabilidades.

El reglamento de ATUDEM sólo es de aplicación a estaciones de esquí alpino, pues las estaciones de esquí de fondo quedan excluidas expresamente en el artículo 1. Por otra parte, cuando el artículo 2 establece las condiciones que han de reunir las estaciones a las que se aplica esta norma, exige la existencia de instalaciones de remonte. El reglamento, por tanto, resulta de imposible aplicación aun en el supuesto de que el explotador de la pista de esquí de fondo perteneciese a ATUDEM.

Parece posible la redacción de un texto equivalente para las estaciones de esquí de fondo. Podrían ser estar integradas en una posible reglamentación de las pistas de esquí de fondo materias tales como la diferenciación entre dominio esquiable y centro turístico o entre zona de dominio esquiable y zonas fuera de la estación, la clasificación de las pistas, el balizamiento, la señalización, la preparación de las pistas, el cierre de pistas, los planes para grandes catástrofes, la organización de la estación, el salvamento y socorro, los derechos y deberes de los usuarios o la utilización de estaciones meteorológicas.

\section{La redacción de un modelo de ordenanza municipal en Francia}

En 1964 el Ministerio del Interior francés hizo pública una disposición municipal tipo para las pistas de esquí alpino. Ante el auge del esquí nórdico, se elaboró otra relativa a la seguridad en las pistas de esquí nórdico, que se sometió a los ayuntamientos por circular del Ministerio del Interior de 9 de julio de 1975.En ella se precisa que sólo se trata de dictar las reglas esenciales para asegurar la seguridad de las personas sin encerrar la práctica del esquí en normas muy estrictas o imponer a los ayuntamientos normas poco razonables sobre infraestructuras. Los ayuntamientos han adaptado este modelo, aceptando su texto pero incorporando otras materias que creían de interés, por ejemplo algunas relacionadas con la seguridad. Se está estudiando una reglamentación similar para los recorridos con raquetas. 
El modelo francés incluye la definición de una pista de esquí de fondo como todo recorrido de nieve balizado, pisado, regularmente mantenido y reservado exclusivamente para la práctica del esquí de fondo. El texto diferencia las diversas formas de traza de pistas, los niveles, la señalización, el balizamiento, su cierre y las prohibiciones de acceso. Igualmente establece la posibilidad de que existan "itinerarios de esquí de fondo", considerados al margen de las pistas de esquí de fondo.

Parece posible en España la redacción de un reglamento para pistas de esquí de fondo o para una pista de esquí de fondo, aunque hasta el momento no exista. Se podría elaborar un modelo de mínimos para una ordenanza municipal, de modo que cada una de las estaciones de esquí de fondo la acogiese e introdujese en ella las modificaciones que creyese necesarias para adaptarla a su particular situación. Tampoco parece existir inconveniente en establecer una reglamentación mínima de estas características, e incluso podrían adoptarse soluciones más ambiciosas que las de los modelos franceses.

\section{El comportamiento del esquiador}

Como complemento de la regulación anterior y ante los problemas planteados por la responsabilidad en los accidentes, la comisión jurídica de la Federación Internacional de Esquí elaboró unas normas de conducta del esquiador de fondo que fueron aprobadas en el congreso celebrado en Argentina en abril de 1977.

\section{Reglas de conducta de los esquiadores de fondo}

Como todo deporte, el esquí de fondo comporta ciertos riesgos y ciertas responsabilidades civiles y penales.

I. Respecto a los otros.

Todo esquiador de fondo debe comportarse de tal manera, que no ponga a los otros en peligro o les pueda causar algún perjuicio.

II. Respecto a la señalización.

El esquiador debe respetar el balizaje y la señalización. Sobre las trazas marcadas, él debe respetar la dirección indicada. 
III. Elección de la traza.

Si hubiera diversas trazas el esquiador debe utilizar la pista de la derecha.Los esquiadores en grupo deben circular en fila por la pista de la derecha.

IV. Adelantamientos.

Los esquiadores que preceden no están obligados a ceder el paso, pero deberían apartarse para dejar paso, si juzgan que pueden hacerlo sin peligro.

El adelantamiento puede efectuarse por la derecha o por la izquierda, por una traza libre o fuera de la traza, pero sin poner en peligro a los otros.

V. Cruces.

En los cruces en las pistas de doble sentido de la marcha, cada uno debe ceder el paso al de la derecha.

Los esquiadores que suben deben dejar la traza a los que descienden.

VI. Bastones.

Los cruces o adelantamientos -tanto para los esquiadores que pasen, como para los que son pasados-deben mantener los bas tones pegados al cuerpo.

VII.Dominio de la velocidad y comportamiento.

Todo esquiador, sobre todo en las pendientes, debe adaptar su velocidad y su comportamiento a sus capacidades personales, así como a las condiciones generales del terreno, del tiempo, de la visi bilidad y de los obstáculos de la pista.

Además deberá conservar un distanciamiento suficiente sobre los esquiadores precedentes.

En último recurso, la caída espontánea puede realizarse para evitar una colisión.

VIII. Estacionamiento.

Las paradas deben efectuarse fuera de la traza.

Aquel que caiga, debe liberar la traza lo más rápidamente posible.

IX. Accidente.

En caso de accidente, toda persona debe prestar socorro.

$X$. Identificación.

Toda persona, testigo o parte responsable o no de un accidente, está obligado a hacer saber su identidad.

De nuevo nos hallamos ante documentos que deberán considerarse en el momento de elaborar las disposiciones de regulación de las pistas de esquí, máxime en este caso al provenir de la comisión jurídica. 


\section{La definición de los Tribunales}

El vacío legal obliga a los jueces y tribunales españoles a realizar un esfuerzo. Al no existir regulación alguna que delimite los perfiles de una estación de esquí los tribunales han debido entrar a definir y describir esos contornos de las actividades empresariales ligadas al esquí. La Audiencia Provincial de Huesca, en su sentencia de 22 de mayo de 1997, se vio obligada a delimitar en qué consiste la estación de esquí de Formigal. Se trata de una estación de esquí alpino pero la definición podría trasladarse a una estación de esquí de fondo.

«SEGUNDO: Es un hecho notorio y pacífico, y como tal se admite en la demanda -hecho primero-, que la sociedad anónima es una empresa mercantil, que explota, con ánimo de lucro, la estación de invierno del mismo nombre, complejo que comprende, básicamente, instalaciones para la práctica del esquí y otras complementarias: remontes mecánicos, pistas acondicionadas y señalizadas, viales, cafeterías y restaurantes, puestos de socorro, etc., y es patente que la gran mayoría de las personas que utilizan los remontes mecánicos lo hacen para después descender esquiando. Es asimismo evidente que la empresa explotadora de la estación realiza una serie de actividades encaminadas a facilitar la práctica del esquí por parte de sus clientes. Concretamente, la empresa tiene las pistas debidamente acondicionadas, numeradas y señalizadas mediante el oportuno balizamiento, y remontes mecánicos y otros medios de transporte para facilitar el acceso a las mismas. El esquí es una actividad deportiva que, por sí misma, entraña ciertos riesgos que conoce y debe asumir quien voluntaria y libremente decide practicarlo, y debe ser consciente, asimismo, de que puede sufrir daños corporales o materiales sin necesidad de que se produzca la acción de un tercero, sea otro practicante del esquí o bien la Empresa que tiene a su favor la concesión para la explotación de determinados parajes naturales, en los que es preciso invertir importantes cantidades de dinero para construir y mantener las instalaciones que son necesarias para desarrollar la aludida actividad.»

Este fundamento jurídico segundo de la sentencia contiene dos partes diferenciadas. La primera, que es la que ahora nos interesa, se aproxima a un concepto que legalmente está por precisar. La segunda resalta el factor de riesgo de la actividad y apunta los posibles responsables de los accidentes que pudieran producirse. 
Un planteamiento similar, especialmente atento a la posición del usuario, se aprecia en la sentencia de la Audiencia Provincial de Cantabria de 10 de diciembre de 1997. Una esquiadora cae de un remonte por arrastre porque la nieve estaba dura o incluso helada en una pista de gran pendiente sin protecciones laterales.

«La entidad que explota la estación invernal debe facilitar a los usuarios de la misma -cuyos servicios éstos abordan mediante la compra de forfait- la utilización segura de los medios con que cuente aquella.Y qué duda cabe que, en un remonte por arrastre con alta pendiente, la falta de redes anticaída constituye una negligencia de la que deriva responsabilidad.»

\section{La seguridad y el socorro}

Los planes de evacuación deben enmarcarse dentro del servicio de Protección Civil. Por lo que respecta a la Comunidad Autónoma de Aragón, según la Orden de 30 de Abril de 1996 que desarrolla la regulación de los Planes de Emergencia de Protección Civil de ámbito municipal, supramunicipal o comarcal y de medidas de fomento, y el Plan Territorial de Protección Civil, aprobado por Decreto 109/1995, de 16 de Mayo, los planes municipales y supramunicipales serán aprobados por el Ayuntamiento u órgano de gobierno de la Entidad Local correspondiente, previa su homologación por la comisión de Protección Civil de Aragón.

En relación con el valor de los instrumentos planificadores, resultan ilustrativas las previsiones que sobre la regulación de la seguridad se han contemplado en diversos Planes de Ordenación de Recursos Naturales de Castilla y León. A título de ejemplo se transcribe el artículo 27 del PORN de La Fuentona aprobado por el Decreto 142/1988, de 16 de julio, de Castilla y León:

«Artículo 27.Seguridad.

Teniendo en cuenta las características privilegiadas que el Espacio Natural contiene para la práctica de deportes de alto riesgo, especialmente el espeleo-buceo, se hace necesario la elaboración de un Plan Especial de Seguridad. 
Se redactará el correspondiente Plan de Seguridad con el objeto de garantizar la seguridad de los visitantes del espacio Natural. Para su elaboración se tendrá en cuenta todas aquellas actividades que pudieran ser causa de accidente, como la práctica de deportes de alto riesgo, así como aquellas otras situaciones del medio que comporten peligrosidad. Dicho Plan preverá los recursos técnicos y humanos necesarios en caso de emergencia. Para ello la Administración del Espacio Natural se coordinará con diferentes organismos como la Guardia Civil, Cruz Roja y Protección Civil.»

\section{La regulación de una pista de esquí de fondo mediante ordenanza municipal}

Cuando se trate de pistas de esquí de fondo cuya titularidad municipal no admita dudas, en cuanto a los edificios y terrenos integrados en la estación, es posible la regulación mediante ordenanza municipal, pero en el caso de que pudieran verse afectados dos o más municipios, sería necesario acudir a otras normas de carácter supramunicipal.

Hay materias que pueden ser reguladas mediante ordenanza municipal. En unos casos no existirán limitaciones de importancia porque se trata de materias sobre las que no existe regulación alguna, pero en otros la ordenanza habrá de adaptarse a la normativa preexistente y de rango superior. En última instancia, la inclusión de unos u otros contenidos en una ordenanza habrá de ser valorada por todas las partes implicadas.

El vacío legal y la necesidad de regular una actividad como el esquí que implica a un importante número de empresarios, trabajadores y consumidores y usuarios no ha culminado en ninguna comunidad autónoma con la redacción de las disposiciones legales que pusiesen orden en el desarrollo de las actividades implicadas en las estaciones de esquí. Aunque el volumen de negocio mayor es el que generan las estaciones de esquí alpino, no resulta inadecuada la regulación de las estaciones de esquí de fondo que tienen una menor importancia desde el punto de vista del desarrollo turístico pero a la vez un menor impacto ambiental.

Nos hallamos ante una instalación turística pero también ante una instalación de carácter deportivo. La Ley 7/1985, de 2 
de Abril, Reguladora de las Bases de Régimen Local, en el artículo 25, incluido en el Capítulo III relativo a las competencias, atribuye éstas a los municipios tanto en lo turístico como en lo deportivo si así lo dispusiesen las legislaciones estatal o autonómica:

«2. El Municipio ejercerá, en todo caso competencias en los términos de la legislación del Estado y de las Comunidades Autónomas, en las siguientes materias:

m) Actividades o instalaciones culturales y deportivas; ocupación del tiempo libre y turismo."

Desde otro punto de vista, el artículo 4 del Reglamento de Bienes de las Entidades Locales establece:

«Son bienes de servicios públicos los destinados directamente al cumplimiento de fines públicos de responsabilidad de las Entidades Locales, tales como Casas Consistoriales ... piscinas y campos de deporte, $y$, en general, cualesquiera otros bienes directamente destinados a la prestación de servicios públicos o administrativos.»

Este Reglamento remite, en su artículo 74, a las normas del Reglamento de Servicios de las Entidades Locales, que ha de regir el uso de los bienes de servicio público. Aunque varios de sus artículos adquieran relieve ante el presente caso, resulta suficientemente significativa la transcripción del artículo 33:

«Las Corporaciones Locales determinarán en la reglamentación de todo servicio que establezcan las modalidades de prestación, situación, deberes y derechos de los usuarios, y, si no se hubieren de desarrollar íntegramente, de quién asumiere la prestación en vez de la Administración.»

Esta breve selección de artículos pone de manifiesto la legalidad y oportunidad de la regulación por ordenanza municipal de una pista de esquí de fondo municipal.

\section{Propuestas de regulación de una pista de esquí de fondo mediante ordenanza municipal}

a) La definición de una estación de esquí de fondo

Una estación de esquí de fondo comprende una serie de instalaciones y servicios coordinados y que se pueden contemplar 
como una unidad.Se podría tratar de definir en qué consiste una estación de esquí de fondo en general, pero en cada caso podría abordarse la definición de la concreta estación de esquí de fondo.

b) Instalaciones y servicios que debe reunir la estación de esquí de fondo

Se trataría de precisar los requisitos mínimos con que debe contar la estación de esquí de fondo en relación con el personal, las comunicaciones, los servicios prestados, los accesos rodados, etc. Estos requisitos, que no se encuentran definidos en la actualidad, debieran establecer tan sólo el mínimo obligatorio.

\section{c) Definición del dominio esquiable}

Parece lógico tratar de delimitar el dominio esquiable. Podría realizarse de manera general mediante el establecimiento de cotas máximas y mínimas pero según los intereses de las partes podrían intentarse delimitaciones más concretas.

\section{d) Clasificación de las pistas}

Es posible intentar la definición de una pista de esquí de fondo pero lo que sí es sin duda necesario es su clasificación según el grado de dificultad. No sería objeto de la ordenanza la fijación de los criterios que sirven para realizar la clasificación pero en este terreno se cuenta con la experiencia suficiente para realizarla con carácter provisional y de conformidad con posibles normas internacionales.

\section{e) Balizamiento de las pistas de esquí de fondo}

El balizamiento se viene practicando por todas las estaciones de esquí de fondo sin que se haya producido la deseable uniformidad. Esta exigencia de uniformidad podría incorporarse a la ordenanza, además de la significación de los colores, los requisitos de las flechas o de las señales de cruce, las de medición de distancias en el recorrido o las de identificación de pistas. La experiencia acumulada habría de servir para elegir entre las diferentes opciones con el mayor acierto.

\section{f) Señalización de la estación de esquí de fondo}

Sería posible establecer, en un marco de búsqueda de uniformidad, otra serie de carteles o letreros referidos de una parte a las prohibiciones, precauciones o peligros y, de otra, a los ser- 
vicios que con carácter obligatorio o facultativo se hayan establecido en la estación de esquí de fondo.

g) Regulación de la apertura y cierre de pistas

Es necesario reglamentar el procedimiento por el cual se lleva a cabo la apertura y el cierre de pistas, dada la trascendencia que tiene el hecho de que una estación cuente o no con servicios para el usuario o se prevean riesgos naturales.

\section{h) Acceso a las pistas}

Es habitual que el acceso a las pistas esté vetado a determinados vehículos, a quienes caminen sin esquíes o a los animales. Deben estar previstas las condiciones de acceso, incluidas las prohibiciones, así como los medios para controlarlo, el posible cerramiento mediante barreras o la utilización de mallas.

\section{i) Vigilancia, control y plan de seguridad}

El hecho de que se trate de una pista municipal hace que sea posible la alusión a los mecanismos de vigilancia y control, dado que en última instancia el Ayuntamiento es el responsable de que se cumpla la normativa en beneficio de los usuarios. El municipio habría de adoptar las medidas oportunas para garantizar la seguridad de todos los visitantes teniendo en cuenta tanto las actividades desarrolladas como los riesgos naturales.

\section{j) Ordenación del tráfico y aparcamiento}

Parece claro que el aparcamiento ha de estar prohibido fuera de las zonas destinadas a este fin. No parece existir problema en establecer la prioridad, por este orden, de los vehículos ligados a los servicios públicos y mantenimiento y los propios usuarios de la pista de esquí de fondo. Tal vez pudiera incorporarse algún precepto acerca de la obligación de conservación y mantenimiento de los accesos por carretera en el marco de las competencias municipales.

\section{k) Organización de competiciones deportivas}

No puede olvidarse que las pistas de esquí de fondo son marco para la celebración de competiciones deportivas que benefician al deporte y a la propia imagen de la zona en que se desarrollan. Será preciso abordar la posibilidad de cerramiento en algunos casos para el buen desarrollo de competiciones 
deportivas de alto nivel, así como prever el despliegue de personas y medios que acompaña a ciertos eventos deportivos.

I) Pistas de esquí de fondo en espacios sometidos a limitaciones

Los pistas trazadas total o parcialmente en Espacios Naturales Protegidos $u$ otros lugares que tengan un especial régimen de protección habrán de someterse a lo dispuesto en su normativa específica.

m) Otros aspectos

Podría estudiarse la incorporación a una ordenanza de la fijación de tasas u otras percepciones, la enseñanza del esquí de fondo, el reconocimiento expreso de los derechos de los usuarios, etc. 